\title{
Modeling of nonlinear propagation in fiber tapers
}

\author{
Lægsgaard, Jesper
}

Published in:

Optical Society of America. Journal B: Optical Physics

Publication date:

2012

Document Version

Publisher's PDF, also known as Version of record

Link back to DTU Orbit

Citation (APA):

Lægsgaard, J. (2012). Modeling of nonlinear propagation in fiber tapers. Optical Society of America. Journal B: Optical Physics, 29(11), 3183-3191.

\section{General rights}

Copyright and moral rights for the publications made accessible in the public portal are retained by the authors and/or other copyright owners and it is a condition of accessing publications that users recognise and abide by the legal requirements associated with these rights.

- Users may download and print one copy of any publication from the public portal for the purpose of private study or research.

- You may not further distribute the material or use it for any profit-making activity or commercial gain

- You may freely distribute the URL identifying the publication in the public portal

If you believe that this document breaches copyright please contact us providing details, and we will remove access to the work immediately and investigate your claim 


\title{
Modeling of nonlinear propagation in fiber tapers
}

\author{
Jesper Lægsgaard \\ DTU•Fotonik, Technical University of Denmark Building 345v, Lyngby DK-280o Kgs, Denmark (jlag@fotonik.dtu.dk)
}

Received July 16, 2012; revised September 28, 2012; accepted October 3, 2012;

posted October 4, 2012 (Doc. ID 172702); published October 30, 2012

A full-vectorial nonlinear propagation equation for short pulses in tapered optical fibers is developed. Specific emphasis is placed on the importance of the field normalization convention for the structure of the equations, and the interpretation of the resulting field amplitudes. Different numerical schemes for interpolation of fiber parameters along the taper are discussed and tested in numerical simulations on soliton propagation and generation of continuum radiation in short photonic-crystal fiber tapers. (c) 2012 Optical Society of America OCIS codes: $\quad 060.4005,060.4370,060.5295,060.5530,190.5650$.

\section{INTRODUCTION}

Nonlinear propagation in tapered fibers has attracted much attention in recent years for several reasons. The development of tapered microwires and nanowires [믈 technology platform that allowed easy interfacing between standard fibers and submicrometer-core devices, and several studies demonstrated broadband supercontinuum generation in such fibers using low-power femtosecond (fs) pulses, typically from Ti:sapphire lasers $[\underline{1}, \underline{4}-\underline{6}]$. More recently, supercontinuum generation from high-power picosecond (ps) pulses has been improved significantly with respect to bandwidth by the use of long slowly tapered photonic crystal fibers (PCFs) [7-10] where the taper is typically obtained directly in the fiber manufacturing step, by varying the parameters of the drawing process. The ps supercontinuum sources provide a broad spectral range with a high spectral power density, but have inferior noise properties compared with fs-generated supercontinuum [11]. In recent years, fiber-based fs laser systems have progressed rapidly [12-14], and recently the generation of Cherenkov radiation from fs pulses in a fully fiberspliced setup was demonstrated [15]. Fully fiber-based fs continuum generation may thus be envisaged in the near future. It is likely that such systems could also benefit from fiber tapers, which would in this case be short, typically a few centimeters, due to the rapid nature of the nonlinear processes induced by fs pulses, and probably obtained using traditional tapering techniques.

Due to the above-mentioned developments, it is of interest to develop accurate methods for detailed modeling of nonlinear propagation in tapered fibers and nanowires. In a recent paper by Vanvincq et al., a detailed derivation of a scalar propagation model was presented, and it was shown that an additional term had to be added to the conventional propagation models for untapered fibers, in order to uphold photon-number conservation [16]. Especially for the case of photonic nanowires, it is of interest to provide a derivation based on the full-vectorial Maxwell equations, as has been done by several authors for the untapered case [17-19]. In this paper, a general propagation equation is derived, based on a field expansion similar to the one used by Kolesik et al. [17]. It is shown that the additional term proposed by Vanvincq et $\mathrm{al}$. comes about due to the adoption of a particular normalization convention, and that it can easily be avoided by choosing a different normalization. It is argued that this alternative normalization leads to a more straightforward interpretation of the time-domain modal amplitudes in terms of propagating power for cases with significant mode profile dispersion. Furthermore, schemes for interpolation of fiber dispersion properties along the taper are discussed and compared by numerical simulations.

\section{FORMALISM}

\section{A. General Propagation Equation}

The starting point is the Maxwell equations with the displacement term separated into a linear term describing the ideal waveguide, and a small perturbation term $\delta \mathbf{P}$ :

$$
\begin{aligned}
& \nabla \times \mathbf{E}=-\mu_{0} \frac{\partial \mathbf{H}}{\partial t}, \\
& \nabla \times \mathbf{H}=\varepsilon_{0} \varepsilon(\mathbf{r}, \omega) \frac{\partial \mathbf{E}}{\partial t}+\frac{\partial \delta \mathbf{P}}{\partial t} .
\end{aligned}
$$

The $\mathbf{E}$ and $\mathbf{H}$ fields are expanded into modal fields:

$$
\begin{gathered}
\mathbf{H}(\mathbf{r}, t)=\frac{1}{\sqrt{2 \pi}} \sum_{m} \int \mathrm{d} \omega A_{m}(z, \omega) \mathbf{h}_{m}(\mathbf{r}, \omega ; t), \\
\mathbf{E}(\mathbf{r}, t)=\frac{1}{\sqrt{2 \pi}} \sum_{m} \int \mathrm{d} \omega A_{m}(z, \omega) \mathbf{e}_{m}(\mathbf{r}, \omega ; t), \\
\mathbf{h}_{m}(\mathbf{r}, \omega ; t)=\mathbf{h}_{m}\left(\mathbf{r}_{\perp}, z, \omega\right) \Phi_{m}(\omega, z, t) \mathbf{e}_{m}(\mathbf{r}, \omega ; t) \\
=\mathbf{e}_{m}\left(\mathbf{r}_{\perp}, z, \omega\right) \Phi_{m}(\omega, z, t), \\
A_{m}(z,-\omega)=A_{m}^{*}(z, \omega) ; \quad \mathbf{e}_{m}\left(\mathbf{r}_{\perp}, z,-\omega\right)=\mathbf{e}_{m}^{*}\left(\mathbf{r}_{\perp}, z, \omega\right),
\end{gathered}
$$

where the $\omega$ integration extends over both positive and negative values, and the $m$-index denotes different mode orders. The $z$-dependent modal fields $\mathbf{h}_{m}\left(\mathbf{r}_{\perp}, z, \omega\right)$ and $\mathbf{e}_{m}\left(\mathbf{r}_{\perp}, z, \omega\right)$ are the modal fields of a straight (nontapered) waveguide whose cross section equals that of the tapered fiber at position $z$. The phase factor $\Phi_{m}(\omega, z, t)$ is given by 


$$
\Phi_{m}(\omega, z, t)=e^{i\left(\omega t-B_{m}(z, \omega)\right)} ; \quad B_{m}(z, \omega)=\int_{0}^{z} \mathrm{~d} z^{\prime} \beta_{m}\left(\omega, z^{\prime}\right),
$$

where $\beta_{m}(\omega, z)$ is the propagation constant of mode $m$ in a straight waveguide calculated with the dielectric distribution at $z$.

The modal fields are orthogonal, and normalized according to

$$
\begin{aligned}
& \int \mathrm{d} \mathbf{r}_{\perp} {\left[\mathbf{e}_{m}\left(\mathbf{r}_{\perp}, \boldsymbol{z}, \omega\right) \times \mathbf{h}_{n}^{*}\left(\mathbf{r}_{\perp}, \boldsymbol{z}, \omega\right)-\mathbf{h}_{m}\left(\mathbf{r}_{\perp}, \boldsymbol{z}, \omega\right) \times \mathbf{e}_{n}^{*}\left(\mathbf{r}_{\perp}, \boldsymbol{z}, \omega\right) n\right]_{z} } \\
&=N_{m}(\omega, z) \delta_{m n} .
\end{aligned}
$$

The choice of normalization $N_{m}(\omega, z)$ is discussed further below.

Due to the $z$-dependence of the transverse modal fields caused by the taper, these fields do not fulfill the linear Maxwell equations exactly, but rather

$$
\begin{aligned}
\nabla \times \mathbf{h}_{m}^{*}(\mathbf{r}, \omega ; t) & =-i \omega \varepsilon_{0} \varepsilon(\mathbf{r}, \omega) \mathbf{e}_{m}^{*}(\mathbf{r}, \omega ; t)+\delta \mathbf{e}_{m}^{*}(\mathbf{r}, \omega ; t) \\
\nabla \times \mathbf{e}_{m}^{*}(\mathbf{r}, \omega ; t) & =i \omega \mu_{0} \mathbf{h}_{m}^{*}(\mathbf{r}, \omega ; t)+\delta \mathbf{h}_{m}^{*}(\mathbf{r}, \omega ; t) \\
\delta \mathbf{e}_{m}(\mathbf{r}, \omega ; t) & =\Phi_{m}(\omega, z, t) \hat{\mathbf{z}} \times \frac{\partial \mathbf{h}_{m}\left(\mathbf{r}_{\perp}, z, \omega\right)}{\partial z} \\
\delta \mathbf{h}_{m}(\mathbf{r}, \omega ; t) & =\Phi_{m}(\omega, z, t) \hat{\mathbf{z}} \times \frac{\partial \mathbf{e}_{m}\left(\mathbf{r}_{\perp}, z, \omega\right)}{\partial z}
\end{aligned}
$$

The fundamental assumption of this work is that $\delta \mathbf{P}$ is sufficiently small that the modal amplitudes $A_{m}(z, \omega)$ are slowly varying compared to $\Phi_{m}(\omega, z, t)$. For silica glass, as well as most other dielectric materials, this is a well-justified assumption, since the optical intensities needed to violate it are typically high above the damage thresholds. It will further be assumed that the length scale of the fiber taper is sufficiently long that $\delta \mathbf{e}, \delta \mathbf{h}$ are also small quantities. Products of these quantities with one another or with $\partial A_{m} / \partial z$ will therefore be neglected in the following derivations.

The derivation of a $1+1 D$ propagation equation proceeds by projecting the Maxwell equations (ㅁ) and (2) onto the modal fields as follows:

$$
\begin{gathered}
\mathbf{e}_{m}^{*}(\mathbf{r}, \omega ; t) \cdot\left[\frac{\partial \delta \mathbf{P}}{\partial t}+\frac{\partial \mathbf{D}}{\partial t}\right]=\mathbf{e}_{m}^{*}(\mathbf{r}, \omega ; t) \cdot \nabla \times \mathbf{H} \\
-\mu_{0} \mathbf{h}_{m}^{*}(\mathbf{r}, \omega ; t) \cdot \frac{\partial \mathbf{H}}{\partial t}=\mathbf{h}_{m}^{*}(\mathbf{r}, \omega ; t) \cdot \nabla \times \mathbf{E}
\end{gathered}
$$

$\Downarrow$

$$
\begin{aligned}
& \mathbf{e}_{m}^{*}(\mathbf{r}, \omega ; t) \cdot\left[\frac{\partial \delta \mathbf{P}}{\partial t}+\frac{\partial \mathbf{D}}{\partial t}\right]= \nabla \cdot\left[\mathbf{H} \times \mathbf{e}_{m}^{*}(\mathbf{r}, \omega ; t)\right]+\mathbf{H} \cdot \nabla \\
& \times \mathbf{e}_{m}^{*}(\mathbf{r}, \omega ; t), \\
&-\mu_{0} \mathbf{h}_{m}^{*}(\mathbf{r}, \omega ; t) \cdot \frac{\partial \mathbf{H}}{\partial t}=\nabla \cdot\left[\mathbf{E} \times \mathbf{h}_{m}^{*}(\mathbf{r}, \omega ; t)\right]+\mathbf{E} \cdot \nabla \times \mathbf{h}_{m}^{*}(\mathbf{r}, \omega ; t) .
\end{aligned}
$$

Using Eqs. (11) and (므), these equations become

$$
\begin{aligned}
\mathbf{e}_{m}^{*}(\mathbf{r}, \omega ; t) \cdot\left[\frac{\partial \delta \mathbf{P}}{\partial t}+\frac{\partial \mathbf{D}}{\partial t}\right]= & \nabla \cdot\left[\mathbf{H} \times \mathbf{e}_{m}^{*}(\mathbf{r}, \omega ; t)\right]+\mathbf{H} \\
& \cdot\left[i \omega \mu_{0} \mathbf{h}_{m}^{*}(\mathbf{r}, \omega ; t)+\delta \mathbf{h}_{m}^{*}(\mathbf{r}, \omega ; t)\right],
\end{aligned}
$$

$$
\begin{aligned}
-\mu_{0} \mathbf{h}_{m}^{*}(\mathbf{r}, \omega ; t) \cdot \frac{\partial \mathbf{H}}{\partial t}= & \nabla \cdot\left[\mathbf{E} \times \mathbf{h}_{m}^{*}(\mathbf{r}, \omega ; t)\right]+\mathbf{E} \\
& \cdot\left[-i \omega \varepsilon_{0} \varepsilon(\mathbf{r}, \omega) \mathbf{e}_{m}^{*}(\mathbf{r}, \omega ; t)+\delta \mathbf{e}_{m}^{*}(\mathbf{r}, \omega ; t)\right]
\end{aligned}
$$

Integrating over time and transverse spatial coordinates, and doing a partial integration assuming that all fields vanish at infinity, one obtains

$$
\begin{aligned}
& i \omega \int \mathrm{d} \mathbf{r}_{\perp} \mathrm{d} t \mathbf{e}_{m}^{*}(\mathbf{r}, \omega ; t)[\delta \mathbf{P}+\mathbf{D}] \\
& =\frac{\partial}{\partial z} \int \mathrm{d} \mathbf{r}_{\perp} \mathrm{d} t\left[\mathbf{H} \times \mathbf{e}_{m}^{*}(\mathbf{r}, \omega ; t)\right]_{z} \\
& +\int \mathrm{d} \mathbf{r}_{\perp} \mathrm{d} t \mathbf{H} \cdot\left[i \omega \mu_{0} \mathbf{h}_{m}^{*}(\mathbf{r}, \omega ; t)+\delta \mathbf{h}_{m}^{*}(\mathbf{r}, \omega ; t)\right] \\
& -i \omega \mu_{0} \int \mathrm{d} \mathbf{r}_{\perp} \mathrm{d} t \mathbf{h}_{m}^{*}(\mathbf{r}, \omega ; t) \cdot \mathbf{H} \\
& =\frac{\partial}{\partial z} \int \mathrm{d} \mathbf{r}_{\perp} \mathrm{d} t\left[\mathbf{E} \times \mathbf{h}_{m}^{*}(\mathbf{r}, \omega ; t)\right]_{z} \\
& \quad+\int \mathrm{d} \mathbf{r}_{\perp} \mathrm{d} t \mathbf{E} \cdot\left[-i \omega \varepsilon_{0} \varepsilon(\mathbf{r}, \omega) \mathbf{e}_{m}^{*}(\mathbf{r}, \omega ; t)\right. \\
& \left.\quad+\delta \mathbf{e}_{m}^{*}(\mathbf{r}, \omega ; t)\right] .
\end{aligned}
$$

The next step is to introduce the modal field expansions [Eqs. (3) and (4)] for the $\mathbf{E}$ and $\mathbf{H}$ fields. The expression of $\delta \mathbf{P}$ in terms of the modal fields is deferred for the moment. This leads to the expressions

$$
\begin{aligned}
& i \omega \int \mathrm{d} \mathbf{r}_{\perp} \mathrm{d} t \mathbf{e}_{m}^{*}(\mathbf{r}, \omega ; t) \delta \mathbf{P} \\
&+i \omega \varepsilon_{0} \sqrt{2 \pi} \sum_{n} \int \mathrm{d} \mathbf{r}_{\perp} A_{n}(z, \omega) \varepsilon(\mathbf{r}, \omega) \mathbf{e}_{n}(\mathbf{r}, \omega ; t) \cdot \mathbf{e}_{m}^{*}(\mathbf{r}, \omega ; t) \\
&= \sqrt{2 \pi} \frac{\partial}{\partial z} \sum_{n} \int \mathrm{d} \mathbf{r}_{\perp}\left[A_{n}(z, \omega) \mathbf{h}_{n}(\mathbf{r}, \omega ; t) \times \mathbf{e}_{m}^{*}(\mathbf{r}, \omega ; t)\right]_{z} \\
&+ \sqrt{2 \pi} \sum_{n} \int \mathrm{d} \mathbf{r}_{\perp}\left[i \omega \mu_{0} A_{n}(z, \omega) \mathbf{h}_{n}(\mathbf{r}, \omega ; t) \cdot \mathbf{h}_{m}^{*}(\mathbf{r}, \omega ; t)\right. \\
&+\left.\mathbf{h}_{n}(\mathbf{r}, \omega ; t) \cdot \delta \mathbf{h}_{m}^{*}(\mathbf{r}, \omega ; t)\right], \\
&-i \omega \mu_{0} \sqrt{2 \pi} \sum_{n} \int \mathrm{d} \mathbf{r}_{\perp} A_{n}(z, \omega) \mathbf{h}_{n}(\mathbf{r}, \omega ; t) \cdot \mathbf{h}_{m}^{*}(\mathbf{r}, \omega ; t) \\
&=\sqrt{2 \pi} \frac{\partial}{\partial z} \sum_{n} \int \mathrm{d} \mathbf{r}_{\perp} A_{n}(z, \omega)\left[\mathbf{e}_{n}(\mathbf{r}, \omega ; t) \times \mathbf{h}_{m}^{*}(\mathbf{r}, \omega ; t)\right]_{z} \\
& \quad+\sqrt{2 \pi} \sum_{n} \int \mathrm{d} \mathbf{r}_{\perp} A_{n}(z, \omega)\left[-i \omega \varepsilon_{0} \varepsilon(\mathbf{r}, \omega) \mathbf{e}_{n}(\mathbf{r}, \omega ; t)\right. \\
&\left.\quad \cdot \mathbf{e}_{m}^{*}(\mathbf{r}, \omega ; t)+\mathbf{e}_{n}(\mathbf{r}, \omega ; t) \cdot \delta \mathbf{e}_{m}^{*}(\mathbf{r}, \omega ; t)\right] .
\end{aligned}
$$

In deriving these equations, it was utilized that the time integral of the product $\Phi_{n}\left(\omega^{\prime}, z, t\right) \Phi_{m}^{*}(\omega, z, t)$ yields a Dirac 
$\delta$-function in $\omega-\omega^{\prime}$ multiplied by $2 \pi$. Subtraction of Eqs. (21) and (22) yields

$$
\begin{aligned}
\frac{i \omega}{\sqrt{2 \pi}} \int & \mathrm{d} \mathbf{r}_{\perp} \mathrm{d} t \mathbf{e}_{m}^{*}(\mathbf{r}, \omega ; t) \delta \mathbf{P} \\
= & -\frac{\partial}{\partial z} A_{m}(z, \omega) N_{m}(\omega, z) \\
& +\sum_{n} A_{n}(z, \omega) \int \mathrm{d} \mathbf{r}_{\perp}\left(\mathbf{h}_{n}(\mathbf{r}, \omega ; t) \cdot \delta \mathbf{h}_{m}^{*}(\mathbf{r}, \omega ; t)-\mathbf{e}_{n}(\mathbf{r}, \omega ; t)\right. \\
& \left.\cdot \delta \mathbf{e}_{m}^{*}(\mathbf{r}, \omega ; t)\right) .
\end{aligned}
$$

Using Eqs. (11) and (12) and the fact that the transverse components of the modal fields $\mathbf{e}_{m}\left(\mathbf{r}_{\perp}, z, \omega\right)$ and $\mathbf{h}_{m}\left(\mathbf{r}_{\perp}, \boldsymbol{z}, \omega\right)$ may be taken purely real, the $n=m$ part of the sum in the second term on the RHS can be rewritten as

$$
\begin{aligned}
& A_{m}(z, \omega) \int \mathrm{d} \mathbf{r}_{\perp}\left[\mathbf{h}_{m}(\mathbf{r}, \omega ; t) \cdot \delta \mathbf{h}_{m}^{*}(\mathbf{r}, \omega ; t)-\mathbf{e}_{m}(\mathbf{r}, \omega ; t) \cdot \delta \mathbf{e}_{m}^{*}(\mathbf{r}, \omega ; t)\right] \\
& \quad=A_{m}(z, \omega) \int \mathrm{d} \mathbf{r}_{\perp} \hat{\mathbf{z}} \cdot\left[\frac{\partial \mathbf{e}_{m}\left(\mathbf{r}_{\perp}, z, \omega\right)}{\partial z} \times \mathbf{h}_{m}^{*}\left(\mathbf{r}_{\perp}, z, \omega\right)\right. \\
& \left.\quad-\frac{\partial \mathbf{h}_{m}\left(\mathbf{r}_{\perp}, z, \omega\right)}{\partial z} \times \mathbf{e}_{m}^{*}\left(\mathbf{r}_{\perp}, z, \omega\right)\right]=\frac{1}{2} A_{m}(z, \omega) \frac{\partial N_{m}}{\partial z}
\end{aligned}
$$

which leads to the propagation equation

$$
\begin{aligned}
N_{m}(\omega, z) \frac{\partial A_{m}}{\partial z}= & -\frac{i \omega}{\sqrt{2 \pi}} \int \mathrm{d} \mathbf{r}_{\perp} \mathrm{d} t \mathbf{e}_{m}^{*}(\mathbf{r}, \omega ; t) \delta \mathbf{P}-\frac{1}{2} A_{m}(z, \omega) \frac{\partial N_{m}}{\partial z} \\
& +\sum_{n \neq m} A_{n}(z, \omega) e^{i\left(B_{m}(\omega, z)-B_{n}(\omega, z)\right)} \\
& \times \int \mathrm{d} \mathbf{r}_{\perp}\left[\mathbf{e}_{n}\left(\mathbf{r}_{\perp}, z, \omega\right) \times \frac{\partial \mathbf{h}_{m}\left(\mathbf{r}_{\perp}, \boldsymbol{z}, \omega\right)}{\partial z}\right. \\
& \left.+\mathbf{h}_{n}\left(\mathbf{r}_{\perp}, \boldsymbol{z}, \omega\right) \times \frac{\partial \mathbf{e}_{m}\left(\mathbf{r}_{\perp}, z, \omega\right)}{\partial z}\right]_{z}
\end{aligned}
$$

The last terms in the equation describe taper-induced coupling between the different eigenmodes of the fiber. If the taper is sufficiently slow, the oscillating nature of the $e^{i\left(B_{m}(\omega, z)-B_{n}(\omega, z)\right)}$ prefactor will suppress these couplings. This is known as the adiabatic limit. If the taper is symmetry-preserving, coupling terms between modes belonging to different symmetry classes, or different polarizations within the same symmetry class, will vanish. Coupling to radiation modes will typically lead to a loss that can be incorporated into the propagation equation in a phenomenological way by adding $z$-dependent imaginary parts to the propagation constants. Thus, the offdiagonal coupling terms may be neglected in many relevant cases, and the derivations will continue without these terms. The diagonal form of Eq. (25) can be written as

$$
\begin{aligned}
N_{m}(\omega, z) \frac{\partial A_{m}}{\partial z}= & -\frac{i \omega}{\sqrt{2 \pi}} \int \mathrm{d} \mathbf{r}_{\perp} \mathrm{d} t \mathbf{e}_{m}^{*}(\mathbf{r}, \omega ; t) \delta \mathbf{P} \\
& -\frac{1}{2} \frac{\partial N_{m}}{\partial z} A_{m}(z, \omega)
\end{aligned}
$$

The last term is easily eliminated by choosing a $z$-independent norm such as $N_{m}(\omega, z)=1$. Note, however, that other choices are in common use. For instance, adopting the scalar approximation, and normalizing the scalar electric field to unity as

$$
\int \mathrm{d} \mathbf{r}_{\perp}\left|E\left(\mathbf{r}_{\perp}\right)\right|^{2}=1,
$$

does not lead to $N_{m}(\omega, z)=1$, but rather $N_{m}(\omega, z)=$ $c \varepsilon_{0} \beta_{m}(\omega, z) / \omega$, and the extra term in the propagation equation must then be taken into account [16].

\section{B. Interpretation of the Amplitudes}

The instantaneous power of the propagating fields can be written as

$$
\begin{aligned}
P(t)= & \frac{1}{2} \int \mathrm{d} \mathbf{r}_{\perp}\left[\mathbf{E}(\mathbf{r}, t) \times \mathbf{H}^{*}(\mathbf{r}, t)-\mathbf{H}(\mathbf{r}, t) \times \mathbf{E}^{*}(\mathbf{r}, t)\right]_{z} \\
= & \frac{1}{4 \pi} \sum_{m n} \int \mathrm{d} \omega_{1} \mathrm{~d} \omega_{2} A_{m}\left(z, \omega_{1}\right) A_{n}^{*}\left(z, \omega_{2}\right) \\
& \times \Phi_{m}\left(\omega_{1}, z, t\right) \Phi_{n}^{*}\left(\omega_{2}, z, t\right) \int \mathrm{d} \mathbf{r}_{\perp}\left[\mathbf{e}_{m}\left(\mathbf{r}_{\perp}, z, \omega_{1}\right)\right. \\
& \left.\times \mathbf{h}_{n}^{*}\left(\mathbf{r}_{\perp}, z, \omega_{2}\right)-\mathbf{h}_{m}\left(\mathbf{r}_{\perp}, z, \omega_{1}\right) \times \mathbf{e}_{n}^{*}\left(\mathbf{r}_{\perp}, \boldsymbol{z}, \omega_{2}\right)\right]_{z} .
\end{aligned}
$$

Neglecting the frequency dependence of the modal fields, and averaging over several optical cycles, this expression becomes

$$
\begin{aligned}
P(t) & =\sum_{m} N_{m}(z)\left|\tilde{A}_{m}(z, t)\right|^{2} \\
\tilde{A}_{m}(z, t) & =\frac{1}{\sqrt{2 \pi}} \int_{0}^{\infty} \mathrm{d} \omega e^{i \omega t} \tilde{A}_{m}(z, \omega) \\
\tilde{A}_{m}(z, \omega) & =A_{m}(z, \omega) e^{-i B_{m}(\omega, z)}
\end{aligned}
$$

Equation (6) has been used to restrict the frequency integral in the definition of $\tilde{A}_{m}(z, t)$ to positive frequencies. Under the stated assumptions, the squared norm of the amplitudes $\tilde{A}_{m}(z, t)$ can then be directly interpreted as a temporal power profile if one chooses $N_{m}(z)=1$. If the mode profiles have a significant frequency variation, this interpretation becomes less straightforward. For the diagonal terms in the sum over modal fields, one can write

$$
\begin{aligned}
\int \mathrm{d} \mathbf{r}_{\perp}\left[\mathbf{e}_{m}\left(\mathbf{r}_{\perp}, z, \omega_{1}\right) \times \mathbf{h}_{m}^{*}\left(\mathbf{r}_{\perp}, z, \omega_{2}\right)-\mathbf{h}_{m}\left(\mathbf{r}_{\perp}, z, \omega_{1}\right)\right. \\
\left.\quad \times \mathbf{e}_{m}^{*}\left(\mathbf{r}_{\perp}, z, \omega_{2}\right)\right]_{z}=\int \mathrm{d} \mathbf{r}_{\perp}\left[\mathbf{e}_{m}\left(\mathbf{r}_{\perp}, z, \omega_{1}\right) \times \mathbf{h}_{m}\left(\mathbf{r}_{\perp}, z, \omega_{2}\right)\right. \\
\left.-\mathbf{h}_{m}\left(\mathbf{r}_{\perp}, z, \omega_{1}\right) \times \mathbf{e}_{m}\left(\mathbf{r}_{\perp}, z, \omega_{2}\right)\right]_{z} \\
=N_{m}\left(\omega_{1}, z\right)+\int \mathrm{d} \mathbf{r}_{\perp}\left[\mathbf{e}_{m}\left(\mathbf{r}_{\perp}, z, \omega_{1}\right) \times \frac{\partial \mathbf{h}_{m}\left(\mathbf{r}_{\perp}, z, \omega_{1}\right)}{\partial \omega_{1}}\right. \\
\left.\quad-\mathbf{h}_{m}\left(\mathbf{r}_{\perp}, z, \omega_{1}\right) \times \frac{\partial \mathbf{e}_{m}\left(\mathbf{r}_{\perp}, z, \omega_{1}\right)}{\partial \omega_{1}}\right]_{z}\left(\omega_{2}-\omega_{1}\right)+O\left(\left(\omega_{2}-\omega_{1}\right)^{2}\right) \\
=N_{m}\left(\omega_{1}, z\right)+\frac{1}{2} \frac{\partial N_{m}\left(\omega_{1}, z\right)}{\partial \omega_{1}}\left(\omega_{2}-\omega_{1}\right)+O\left(\left(\omega_{2}-\omega_{1}\right)^{2}\right) .
\end{aligned}
$$

The first equality stems from the fact that the transverse components of the modal fields $\mathbf{e}_{m}\left(\mathbf{r}_{\perp}, z, \omega\right)$ and $\mathbf{h}_{m}\left(\mathbf{r}_{\perp}, z, \omega\right)$ may be taken real. It is seen that if a frequency-independent $N_{m}(\omega, z)$ is chosen, such as $N_{m}(\omega, z)=1$, the interpretation of $\left|\tilde{A}_{m}(z, t)\right|^{2}$ as temporal modal power profiles holds to first order in the frequency dispersion of the mode profiles, provided that off-diagonal terms can be neglected. This will often be the case when modes belong to different symmetry classes, 
or between different polarization states of modes of the same symmetry.

Regardless of mode profile dispersion, the total pulse energy is given by

$$
E_{p}=\int \mathrm{d} t P(t)=\frac{1}{2} \sum_{m} \int \mathrm{d} \omega\left|\tilde{A}_{m}(z, \omega)\right|^{2} N_{m}(\omega, z),
$$

meaning that for $N_{m}(\omega, z)=1$ the positive-frequency normsquared amplitudes $A_{m}(z, \omega)$ integrate directly to the total pulse energy.

In summary, the choice of a frequency- and $z$-independent norm, such as $N_{m}(\omega, z)=1$, leads to a simplification of the propagation equation, as well as a more straightforward interpretation of the field envelope as an instantaneous power density. In the following subsection, the development of the propagation equation will be finalized, by considering a specific form for the nonlinear term, using this normalization convention.

\section{Nonlinear Polarization}

For an isotropic material such as silica glass, the vector components of the third-order nonlinear polarization can be written as [20]

$$
P_{i}(\mathbf{r})=\sum_{j k l} E_{j}(\mathbf{r}, t) \chi^{(3)}(\mathbf{r}) \int \mathrm{d} t^{\prime} r_{i j k l}\left(t-t^{\prime}\right) E_{k}\left(\mathbf{r}, t^{\prime}\right) E_{l}\left(\mathbf{r}, t^{\prime}\right)
$$

The response tensor $r$ has an electronic contribution that is proportional to $\delta\left(t-t^{\prime}\right) \delta_{i j k l}$, and a delayed-response (Raman) term, which can be written as

$$
r_{i j k l}^{R}(t)=a(t) \delta_{i j} \delta_{k l}+\frac{1}{2} b(t)\left(\delta_{i k} \delta_{j l}+\delta_{i l} \delta_{j k}\right)
$$

Experimental data indicate that $b(t) \ll a(t)$, over most of the frequency spectrum of these functions. Neglecting $b(t)$, Eq. (32) can be recast as

$$
\delta \mathbf{P}(\mathbf{r}, t)=\varepsilon_{0} \chi^{(3)}(\mathbf{r}) \mathbf{E}(\mathbf{r}, t) \int \mathrm{d} t^{\prime} \tilde{R}\left(\mathbf{r}, t-t^{\prime}\right)\left|E\left(\mathbf{r}, t^{\prime}\right)\right|^{2} .
$$

Note that this form is also exact if the direction of the electric-field vector at any point in the fiber cross section is time-independent, e.g., for a linearly polarized single mode. The formalism of this paper will be developed from Eq. (34).

Inserting the modal expansion for $\mathbf{E}$, one obtains

$$
\begin{aligned}
& \frac{i \omega}{\sqrt{2 \pi}} \int \mathrm{d} \mathbf{r}_{\perp} \mathrm{d} t \mathbf{e}_{m}^{*}(\mathbf{r}, \omega ; t) \cdot \delta \mathbf{P}(\mathbf{r}, t) \\
& =\frac{i \omega \varepsilon_{0}}{\sqrt{2 \pi}} \sum_{n p q} \int \mathrm{d} \omega_{1-2} \tilde{A}_{n}\left(z, \omega_{1}\right) \tilde{A}_{p}^{*}\left(z, \omega_{2}\right) \tilde{A}_{q}\left(z, \omega-\omega_{1}\right. \\
& \left.\quad+\omega_{2}\right) e^{i B_{m}(\omega, z)} \times \int \mathrm{d} \mathbf{r}_{\perp} \chi^{(3)}(\mathbf{r}) \tilde{R}\left(\mathbf{r}, \omega-\omega_{1}\right) \mathbf{e}_{m}^{*}\left(\mathbf{r}_{\perp}, z, \omega\right) \\
& \quad \cdot \mathbf{e}_{n}\left(\mathbf{r}_{\perp}, z, \omega_{1}\right) \mathbf{e}_{p}^{*}\left(\mathbf{r}_{\perp}, z, \omega_{2}\right) \cdot \mathbf{e}_{q}\left(\mathbf{r}_{\perp}, z, \omega-\omega_{1}+\omega_{2}\right),
\end{aligned}
$$

after a few manipulations. Using this form, with the frequency integrals extending over both positive and negative frequen- cies, in the propagation equation results in a quite general formalism capable of describing a variety of phenomena including third-harmonic generation. However, for reasons of numerical efficiency it is often desirable to restrict the formalism to consider only positive frequencies, by utilizing Eq. (6). Assuming that the spectrum of the optical pulse is sufficiently narrow that Eq. (35) does not have contributions from negative values of $\omega_{1}, \omega_{2}$ as long as $\omega$ is positive, one can recast this expression into the form

$$
\begin{aligned}
& \frac{i \omega}{\sqrt{2 \pi}} \int \mathrm{d} \mathbf{r}_{\perp} \mathrm{d} t \mathbf{e}_{m}^{*}(\mathbf{r}, \omega ; t) \cdot \delta \mathbf{P}(\mathbf{r}, t) \\
& \approx \frac{i \omega \varepsilon_{0}}{\sqrt{2 \pi}} \sum_{n p q} \int_{+} \mathrm{d} \omega_{1-2} \tilde{A}_{n}\left(z, \omega_{1}\right) \tilde{A}_{p}^{*}\left(z, \omega_{2}\right) \tilde{A}_{q}\left(z, \omega-\omega_{1}+\omega_{2}\right) e^{i B_{m}(\omega, z)} \\
& \quad \times \int \mathrm{d} \mathbf{r}_{\perp} \chi^{(3)}(\mathbf{r})\left[2 \tilde{R}\left(\mathbf{r}, \omega-\omega_{1}\right) \mathbf{e}_{m}^{*}\left(\mathbf{r}_{\perp}, z, \omega\right)\right. \\
& \quad \cdot \mathbf{e}_{n}\left(\mathbf{r}_{\perp}, z, \omega_{1}\right) \mathbf{e}_{p}^{*}\left(\mathbf{r}_{\perp}, z, \omega_{2}\right) \cdot \mathbf{e}_{q}\left(\mathbf{r}_{\perp}, z, \omega-\omega_{1}+\omega_{2}\right) \\
& \quad+\tilde{R}\left(\mathbf{r}, \omega+\omega_{2}\right) \mathbf{e}_{m}^{*}\left(\mathbf{r}_{\perp}, z, \omega\right) \cdot \mathbf{e}_{n}^{*}\left(\mathbf{r}_{\perp}, z, \omega_{2}\right) \mathbf{e}_{p}\left(\mathbf{r}_{\perp}, z, \omega_{1}\right) \\
& \left.\quad \cdot \mathbf{e}_{q}\left(\mathbf{r}_{\perp}, z, \omega-\omega_{1}+\omega_{2}\right)\right] .
\end{aligned}
$$

The response function can be written as the sum of an instantaneous (Kerr) term, and a delayed term arising from Raman scattering:

$$
\tilde{R}(\mathbf{r}, \omega)=\left(1-f_{R}\right)+f_{R} R(\mathbf{r}, \omega) .
$$

For fiber materials one can assume that $R\left(\mathbf{r}, \omega+\omega_{2}\right)$ is negligible, because phonon energies are much smaller than the optical energies of photons. The nonlinear polarization then becomes

$$
\begin{aligned}
& \frac{i \omega}{\sqrt{2 \pi}} \int \mathrm{d} \mathbf{r}_{\perp} \mathrm{d} t \mathbf{e}_{m}^{*}(\mathbf{r}, \omega ; t) \cdot \delta \mathbf{P}(\mathbf{r}, t) \\
& \approx \frac{i \omega \varepsilon_{0}}{\sqrt{2 \pi}} \sum_{n p q} \int_{+} \mathrm{d} \omega_{1-2} \tilde{A}_{n}\left(z, \omega_{1}\right) \tilde{A}_{p}^{*}\left(z, \omega_{2}\right) \tilde{A}_{q}\left(z, \omega-\omega_{1}+\omega_{2}\right) e^{i B_{m}(\omega, z)} \\
& \quad \times\left[\left(1-f_{R}\right)\left(2 K_{m n p q}^{(1)} \tilde{R}\left(\mathbf{r}, \omega-\omega_{1}\right)+K_{m n p q}^{(2)}\right)\right. \\
& \left.\quad+2 f_{R} R\left(\mathbf{r}, \omega-\omega_{1}\right) K_{m n p q}^{(1)}\right]
\end{aligned}
$$

$$
\begin{aligned}
K_{m n p q}^{(1)}= & \int \mathrm{d} \mathbf{r}_{\perp} \chi^{(3)}(\mathbf{r}) \mathbf{e}_{m}^{*}\left(\mathbf{r}_{\perp}, \boldsymbol{z}, \omega\right) \cdot \mathbf{e}_{n}\left(\mathbf{r}_{\perp}, z, \omega_{1}\right) \mathbf{e}_{p}^{*}\left(\mathbf{r}_{\perp}, \boldsymbol{z}, \omega_{2}\right) \\
& \cdot \mathbf{e}_{q}\left(\mathbf{r}_{\perp}, \boldsymbol{z}, \omega-\omega_{1}+\omega_{2}\right),
\end{aligned}
$$

$$
\begin{aligned}
K_{m n p q}^{(2)}= & \int \mathrm{d} \mathbf{r}_{\perp} \chi^{(3)}(\mathbf{r}) \mathbf{e}_{m}^{*}\left(\mathbf{r}_{\perp}, \boldsymbol{z}, \omega\right) \cdot \mathbf{e}_{n}^{*}\left(\mathbf{r}_{\perp}, \boldsymbol{z}, \omega_{2}\right) \mathbf{e}_{p}\left(\mathbf{r}_{\perp}, \boldsymbol{z}, \omega_{1}\right) \\
& \cdot \mathbf{e}_{q}\left(\mathbf{r}_{\perp}, \boldsymbol{z}, \omega-\omega_{1}+\omega_{2}\right) .
\end{aligned}
$$

Inserting this expression into the propagation equation (25), one obtains a fairly general description of nonlinear propagation phenomena, excluding third-harmonic generation. However, the appearance of the complicated overlap integrals $K_{1}$ and $K_{2}$ makes the equation numerically cumbersome. An approach for simplification has been discussed in an earlier publication [21], and will be illustrated in the next subsection for the special case of a single-mode field expansion and a single-material fiber. 


\section{Formulation for a Single Mode}

The formulation of a propagation equation in terms of a single-polarization single-mode expansion is highly desirable from the point of view of numerical efficiency, and is often sufficient to obtain a good description of actual experiments. In the following, the state index on the field amplitudes will therefore be dropped, and it will also be implicitly understood that frequency integrals are to be evaluated over the positive half-axis only. Furthermore, it will be assumed that the fiber consists of one nonlinear material only, possibly with additional sections of air/vacuum where the nonlinear coefficient of the material is negligible. The generalization to multiple nonlinear materials is sometimes necessary (for instance when modeling nonlinear propagation in hollow-core photonic bandgap fibers), and is straightforward to formulate [22].

With only a single term in the modal expansion, the combined equations (25) and (하) become

$$
\begin{aligned}
\frac{\partial A(z, \omega)}{\partial z}= & -\frac{i \omega \varepsilon_{0}}{\sqrt{2 \pi}} \int \mathrm{d} \omega_{1-2} \tilde{A}\left(z, \omega_{1}\right) \tilde{A}^{*}\left(z, \omega_{2}\right) \tilde{A}\left(z, \omega-\omega_{1}+\omega_{2}\right) e^{i B(\omega, z)} \\
& \times\left[\left(1-f_{R}\right)\left(2 K^{(1)} \tilde{R}\left(\mathbf{r}, \omega-\omega_{1}\right)+K^{(2)}\right)\right. \\
& \left.+2 f_{R} R\left(\mathbf{r}, \omega-\omega_{1}\right) K^{(1)}\right] .
\end{aligned}
$$

To obtain a numerically tractable equation, the $K^{(1)}$ and $K^{(2)}$ coefficients will be approximated by the expressions

$$
\begin{aligned}
K^{(1)} \approx & \frac{\mu_{0} \chi^{(3)}}{4 n_{m}^{2} \varepsilon_{0}}\left[A_{\mathrm{eff}}(z, \omega) A_{\mathrm{eff}}\left(z, \omega_{1}\right) A_{\mathrm{eff}}\left(z, \omega_{2}\right)\right. \\
& \left.\times A_{\mathrm{eff}}\left(z, \omega-\omega_{1}+\omega_{2}\right)\right]^{-1 / 4}, \\
K^{(2)} \approx & \frac{\mu_{0} \chi^{(3)}}{4 n_{m}^{2} \varepsilon_{0}}\left[A_{\mathrm{eff}}^{\prime}(z, \omega) A_{\mathrm{eff}}^{\prime}\left(z, \omega_{1}\right) A_{\mathrm{eff}}^{\prime}\left(z, \omega_{2}\right)\right. \\
& \left.\times A_{\mathrm{eff}}^{\prime}\left(z, \omega-\omega_{1}+\omega_{2}\right)\right]^{-1 / 4}, \\
A_{\mathrm{eff}}(z, \omega)= & \frac{\mu_{0}\left[\operatorname{Re}\left(\int \mathrm{d} \mathbf{r}_{\perp} \mathbf{e}\left(\mathbf{r}_{\perp}, z, \omega\right) \times \mathbf{h}^{*}\left(\mathbf{r}_{\perp}, z, \omega\right)\right)\right]^{2}}{n_{m}^{2} \varepsilon_{0} \int_{m} \mathrm{~d} \mathbf{r}_{\perp}\left|\mathbf{e}^{*}\left(\mathbf{r}_{\perp}, z, \omega\right)\right|^{4}},
\end{aligned}
$$

$$
\begin{aligned}
& A_{\mathrm{eff}}^{\prime}(\boldsymbol{z}, \omega) \\
& \quad=\frac{\mu_{0}\left[\operatorname{Re}\left(\int \mathrm{d} \mathbf{r}_{\perp} \mathbf{e}\left(\mathbf{r}_{\perp}, \boldsymbol{z}, \omega\right) \times \mathbf{h}^{*}\left(\mathbf{r}_{\perp}, \boldsymbol{z}, \omega\right)\right)\right]^{2}}{n_{m}^{2} \varepsilon_{0} \int_{m} \mathrm{~d} \mathbf{r}_{\perp} \mathbf{e}^{*}\left(\mathbf{r}_{\perp}, z, \omega\right) \cdot \mathbf{e}^{*}\left(\mathbf{r}_{\perp}, z, \omega\right) \mathbf{e}\left(\mathbf{r}_{\perp}, z, \omega\right) \cdot \mathbf{e}\left(\mathbf{r}_{\perp}, z, \omega\right)}
\end{aligned}
$$

The notation $\int_{m} \mathrm{~d} \mathbf{r}_{\perp}$ indicates integration over that part of the fiber cross section that consists of nonlinear material with refractive index $n_{m}$, and $\chi^{(3)}$ denotes its nonlinear coefficient. The numerical advantage of this decomposition is that the double-frequency integral in Eq. (41) can now be evaluated efficiently by a sequence of fast Fourier transform convolutions. It has been shown, albeit for a scalar problem, that this approximation compares very favorably with results of more accurate evaluations of $K^{(1)}$, and that several analytical arguments of a general nature favor this approach [21], which will therefore be adopted without further justification in this paper. Equation (41) can now be rewritten

$$
\begin{aligned}
\frac{\partial A(z, \omega)}{\partial z}= & i \omega \frac{\mu_{0} \chi^{(3)}}{4 n_{m}^{2} \sqrt{2 \pi}} \int \mathrm{d} \omega_{1-2}\left\{2\left[A_{\mathrm{eff}}(z, \omega)\right]^{-1 / 4} \bar{A}\left(z, \omega_{1}\right)\right. \\
& \times \bar{A}^{*}\left(z, \omega_{2}\right) \bar{A}\left(z, \omega-\omega_{1}+\omega_{2}\right) e^{i B(\omega, z)}\left[\left(1-f_{R}\right)\right. \\
& \left.+f_{R} R\left(\mathbf{r}, \omega-\omega_{1}\right)\right]+\left[A_{\mathrm{eff}}^{\prime}(z, \omega)\right]^{-1 / 4} \bar{A}^{\prime}\left(z, \omega_{1}\right) \\
& \left.\times \bar{A}^{\prime *}\left(z, \omega_{2}\right) \bar{A}^{\prime}\left(z, \omega-\omega_{1}+\omega_{2}\right) e^{i B(\omega, z)}\left(1-f_{R}\right)\right\}
\end{aligned}
$$

$$
\bar{A}(z, \omega)=\frac{\tilde{A}(z, \omega)}{\left[A_{\mathrm{eff}}(z, \omega)\right]^{1 / 4}} ; \quad \bar{A}^{\prime}(z, \omega)=\frac{\tilde{A}(z, \omega)}{\left[A_{\mathrm{eff}}^{\prime}(z, \omega)\right]^{1 / 4}}
$$

The difference between $A_{\text {eff }}(z, \omega)$ and $A_{\text {eff }}^{\prime}(z, \omega)$ was pointed out by Afshar, but was overlooked in some earlier work $[\underline{21}, \underline{22}]$. Its origin can be understood by noting that the transverse field components of the eigenmode may be taken real, which then implies a purely imaginary $z$-component. Therefore, if the $z$-component has a substantial magnitude, $A_{\text {eff }}(z, \omega)$ and $A_{\text {eff }}^{\prime}(z, \omega)$ may differ significantly. This will be the case for waveguides with small cores and large index contrast. In Fig. 1 the relative difference between the two areas is plotted for a silica nanowire, as a function of $d / \lambda$ where $d$ is the nanowire diameter. It is seen that the relative difference is mostly small, but can reach values around 0.4 for
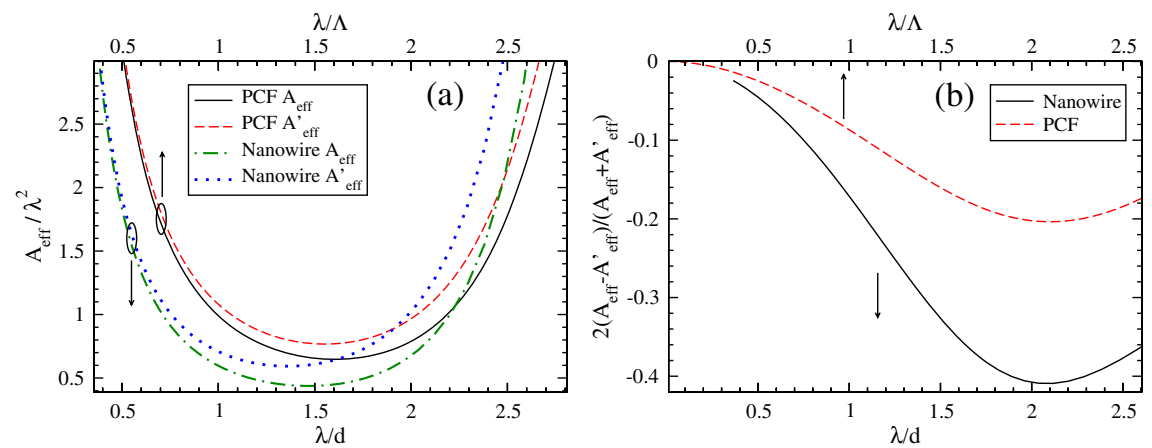

Fig. 1. (Color online) (a) $A_{\text {eff }}$ and $A_{\text {eff }}^{\prime}$ as a function of normalized wavelengths for a silica nanowire and a PCF. (b) Relative difference between $A_{\text {eff }}$ and $A_{\text {eff }}^{\prime}$ in the two cases. The wavelength is normalized to nanowire diameter $d$, and PCF pitch $\Lambda$, respectively. 
sufficiently small nanowire diameters. Since the difference between $1 / A_{\text {eff }}(z, \omega)$ and $1 / A_{\text {eff }}^{\prime}(z, \omega)$ is weighted by $1 / 3$ in the propagation equation, the approximation $A_{\text {eff }}^{\prime}(z, \omega) \approx$ $A_{\text {eff }}(z, \omega)$ can at most lead to an inaccuracy of $\sim 14 \%$ in the estimation of the Kerr nonlinearity. Also shown in Fig. 1 is the corresponding curve for a $\mathrm{PCF}$, with a relative hole diameter of 0.8. In this case, the relative difference between $A_{\text {eff }}(z, \omega)$ and $A_{\text {eff }}^{\prime}(z, \omega)$ does not exceed 0.2 . If the approximation $A_{\text {eff }}^{\prime}(z, \omega) \approx A_{\text {eff }}(z, \omega)$ is accepted, Eq. (46) becomes

$$
\begin{aligned}
\frac{\partial A(z, \omega)}{\partial z}= & i \omega \frac{3 \mu_{0} \chi^{(3)}}{4 n_{m}^{2} \sqrt{2 \pi}} \int \mathrm{d} \omega_{1-2}\left[A_{\mathrm{eff}}(z, \omega)\right]^{-1 / 4} \bar{A}\left(z, \omega_{1}\right) \\
& \times \bar{A}^{*}\left(z, \omega_{2}\right) \bar{A}\left(z, \omega-\omega_{1}+\omega_{2}\right) e^{i B(\omega, z)} \\
& \times\left[\left(1-f_{R}\right)+\frac{2}{3} f_{R} R\left(\mathbf{r}, \omega-\omega_{1}\right)\right] \\
= & i \frac{\omega}{c} n_{2} \sqrt{2 \pi} \int \mathrm{d} \omega_{1-2}\left[A_{\mathrm{eff}}(z, \omega)\right]^{-1 / 4} \bar{A}\left(z, \omega_{1}\right) \bar{A}^{*}\left(z, \omega_{2}\right) \\
& \times \bar{A}\left(z, \omega-\omega_{1}+\omega_{2}\right) e^{i B(\omega, z)}\left[\left(1-f_{R}\right)+\frac{2}{3} f_{R} R\left(\mathbf{r}, \omega-\omega_{1}\right)\right] \\
= & i \frac{\omega}{c} \tilde{n}_{2} \sqrt{2 \pi} \int \mathrm{d} \omega_{1-2}\left[A_{\mathrm{eff}}(z, \omega)\right]^{-1 / 4} \bar{A}\left(z, \omega_{1}\right) \\
& \times \bar{A}^{*}\left(z, \omega_{2}\right) \bar{A}\left(z, \omega-\omega_{1}+\omega_{2}\right) e^{i B(\omega, z)}\left[\left(1-\tilde{f}_{R}\right)\right. \\
& \left.+\tilde{f}_{R} R\left(\mathbf{r}, \omega-\omega_{1}\right)\right], \\
n_{2}= & \frac{3 \chi^{(3)}}{4 \varepsilon_{0} c n_{m}^{2}} ; \quad \tilde{n}_{2}=n_{2}\left(1-\frac{f_{R}}{3}\right) ; \quad \tilde{f}_{R}=\frac{2 f_{R}}{3\left(1-\frac{f_{R}}{3}\right)} .
\end{aligned}
$$

The last identity shows that the weighting factors of Kerr and Raman terms can be expressed as either $\left(1-f_{R}\right)$ and $(2 / 3) f_{R}$ or $\left(1-\tilde{f}_{R}\right)$ and $\tilde{f}_{R}$ with a suitable redefinition of $n_{2}$. In the literature, both conventions have been used by various authors. The equivalence of the two approaches implies one should use that which is consistent with the analysis leading to the nonlinear parameters used. Blow and Wood [23] deduced $f_{R}=0.3$ by comparing to experimental data for the Raman gain. A commonly used value for $\tilde{f}_{R}$ is 0.18 [24], which is roughly consistent with $\tilde{f}_{R}=(2 / 3) f_{R}$. The resulting difference between $n_{2}$ and $\tilde{n_{2}}$ is then around $10 \%$, which is comparable to the spread in published $n_{2}$ values.

\section{E. Interpolation of Fiber Parameters}

A final question pertinent to the modeling of tapered fibers is how to determine $\beta$ and $A_{\text {eff }}$ as a function of $z$. A straightforward procedure is to calculate dispersion and effective areas for a number of fiber structures representing intermediate points of the taper, and linearly interpolate $\beta$ and $A_{\text {eff }}$ between the calculated values. As an example, for a PCF taper where the structure is assumed to be preserved except for a variation in the pitch $\Lambda$ (the center-to-center distance between neighboring airholes in the cladding), one would calculate the PCF properties for the initial and final $\Lambda$ values, as well as some intermediate values. This approach is straightforwardly generalized to situations where both $\Lambda$ and the normalized hole diameter $d / \Lambda$ vary along the taper. On the other hand, if only $\Lambda$ is varying, the effective-area curves can be calculated at any point along the taper by utilizing the scaling properties of Maxwell's equations. The dispersion curve is less straightforward, due to the importance of material dispersion, which breaks this scale invariance. However, it has been shown [25] that the dispersion of PCFs with varying $\Lambda$ can be predicted quite accurately from the result of a single calculation, with constant refractive indices, of $\beta(\omega)$, group velocity, and the fraction of field energy residing in the dispersive material. This can be achieved through a first-order perturbative treatment of material dispersion effects. Results are found to be highly accurate in a broad frequency range around the frequency for which the chosen material refractive index in the reference calculation is correct. In this work, this approach was chosen as a reference against which linear interpolations of dispersion and area parameters with a varying number of interpolation points are benchmarked. For an efficient implementation of the perturbative scheme, the abovementioned parameters were calculated at a relatively small number of frequency points $(\sim 200)$, and at each taper point, $\beta(\omega)$ and its first and second derivatives, $\beta_{1}(\omega), \beta_{2}(\omega)$, were calculated using the perturbative technique. The $\beta(\omega)$ curve can then be interpolated onto the much finer (several thousand points) grid used for the nonlinear propagation calculations, by integrating the equations

$$
\frac{d \beta}{d \omega}=\beta_{1}(\omega) ; \quad \frac{d \beta_{1}}{d \omega}=\beta_{2}(\omega),
$$

between points on the coarse mesh. This approach is sufficiently efficient that it can be implemented at every step of the (Buliersch-Stoer) integration routine used for solving the nonlinear propagation equation, with insignificant computational overhead.

\section{NUMERICAL RESULTS}

In the numerical tests, a PCF having a relative hole diameter of $d / \Lambda=0.8$ was considered. Dispersion and effective areas for a constant refractive index were calculated in normalized units using the plane-wave-based freely available software package MIT Photonic Bands. The difference between $A_{\text {eff }}$ and $A_{\text {eff }}^{\prime}$ was taken into account; i.e., Eq. (46) rather than Eq. (48) was solved. The difference between results of the two equations was found to be minor for the cases considered. For the calculations using linear interpolations of fiber

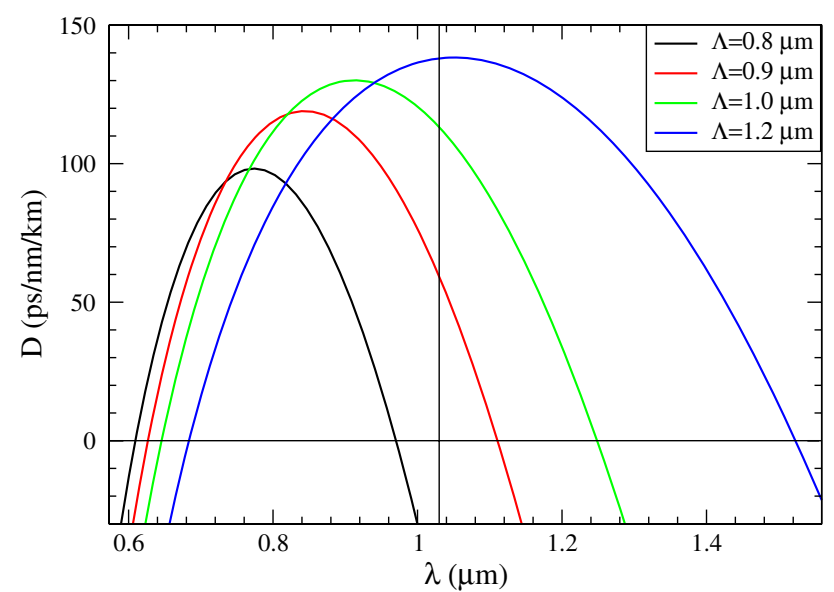

Fig. 2. (Color online) Dispersion curves for the PCF investigated at small values of $\Lambda$. The vertical line indicates the wavelength of the initial pulse, $\lambda=1.03 \mu \mathrm{m}$. 

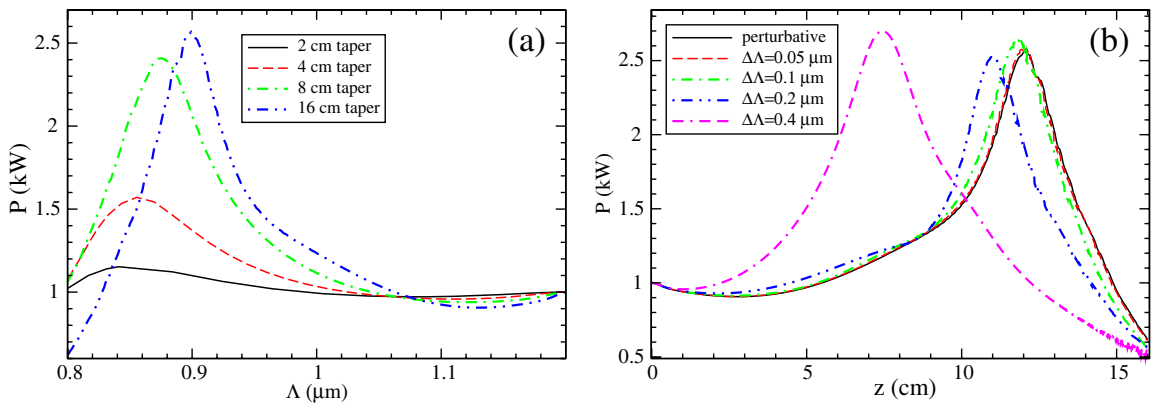

Fig. 3. (Color online) (a) Soliton peak power versus pitch $(\Lambda)$ of the tapered fiber for different taper rates. (b) Soliton peak power versus distance for a $16 \mathrm{~cm}$ taper, using either perturbative calculation of the dispersion properties, or linear interpolation between parameters for a finite number of $\Lambda$-values.

parameters, the $\beta(\omega)$ curve was calculated at specific values of $\Lambda$ using the perturbative approach, so that the (slight) inaccuracies of this method do not influence the benchmarking of the linear interpolation scheme.

As a first test case, the propagation of a single soliton in a tapered PCF with small values of $\Lambda$ was investigated. An ideal fundamental soliton with a peak power of $1 \mathrm{~kW}$ was launched at a wavelength of $1.03 \mu \mathrm{m}$, into a tapered PCF with an initial $\Lambda=1.2 \mu \mathrm{m}$. The fiber was linearly down-tapered to $\Lambda=$ $0.8 \mu \mathrm{m}$ over a distance that was varied from 2 to $16 \mathrm{~cm}$. The evolution of the PCF dispersion curve in this $\Lambda$-range is illustrated in Fig. 2. As the fiber is down-tapered, the dispersion, as well as the effective area, at the soliton wavelength will decrease, leading to soliton compression. Eventually, the long-wavelength zero-dispersion point crosses the soliton wavelength, and the soliton is pushed toward lower frequencies while at the same time losing much of its power to dispersive waves at longer wavelengths.

In Fig. 3(a), the evolution of the pulse peak power along the taper is shown, as calculated using the perturbative approach for modeling of the tapered fiber properties, for various taper lengths. To facilitate comparison, the peak power is plotted versus the local value of $\Lambda$, rather than $z$. For a short taper, the soliton cannot follow the evolution of fiber parameters adiabatically, and no large compression is obtained. For taper lengths of 8 and $16 \mathrm{~cm}$, a compression factor of around 2.5 on the peak power is obtained, just before the zero-dispersion point crosses the soliton wavelength. In Fig. $\underline{3(b)}$, a

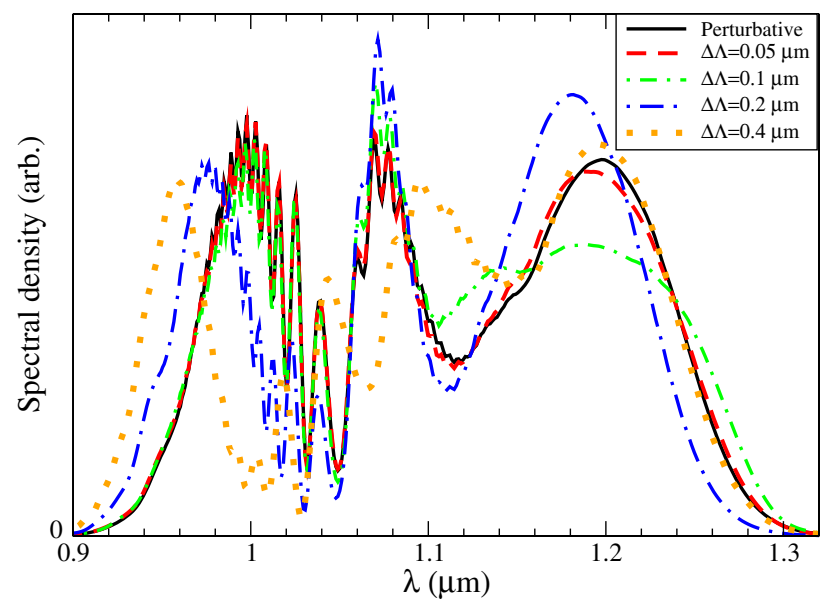

Fig. 4. (Color online) Final spectra for soliton propagation in a tapered fiber of $16 \mathrm{~cm}$, for the different fiber interpolation schemes. comparison of the perturbative calculation with various linear interpolation calculations is shown for a taper length of $16 \mathrm{~cm}$. In the most refined of these calculations, the fiber dispersion was interpolated between 9 fibers, with a $0.05 \mu \mathrm{m}$ difference in $\Lambda$ between each fiber, denoted as $\Delta \Lambda$ in the figure. The other extreme is a calculation with direct linear interpolation of $\beta$ and $A_{\text {eff }}$ between the values at $\Lambda=0.8 \mu \mathrm{m}$, and $\Lambda=1.2 \mu \mathrm{m}$. It is evident that a reasonable computational accuracy requires $\Delta \Lambda=0.1 \mu \mathrm{m}$ or smaller. A similar conclusion emerges from the final spectra (at $\Lambda=0.8 \mu \mathrm{m}$ ) for the different interpolation schemes, which are shown in Fig. 4 .

In these calculations, the photon number was conserved to a level of approximately $10^{-10}$, corresponding to a deviation of less than one photon between initial and final pulses (note that initial quantum noise and spontaneous Raman scattering was not included in the calculations). This confirms that the formalism developed conserves the photon number without the need for correction terms.

As a second test of an experimentally relevant problem, continuum generation from a short high-power pulse was investigated. An input pulse with a Gaussian shape, a peak power of $20 \mathrm{~kW}$, a central wavelength of $1.03 \mu \mathrm{m}$, and a temporal FWHM of 50 fs was chosen for this example. The initial pitch of the PCF was taken to be $4 \mu \mathrm{m}$, which was tapered down to $1 \mu \mathrm{m}$ over a distance of $5 \mathrm{~cm}$. A spectrogram of the final pulse after $5 \mathrm{~cm}$ propagation is shown in Fig. $\underline{5}$. As the pulse propagates, it initially undergoes compression and breakup, with the formation of a strong red-shifted soliton, and dispersive waves at shorter wavelengths. Some of these dispersive waves are emitted in front of the soliton,

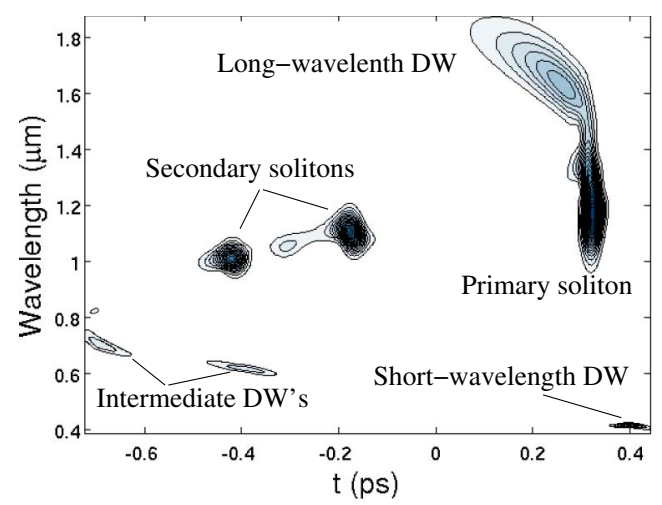

Fig. 5. (Color online) Spectrogram of fs pulse propagated in $5 \mathrm{~cm}$ fiber taper, from $\Lambda=4 \mu \mathrm{m}$ to $\Lambda=1 \mu \mathrm{m}$. The main features of the broken-up pulse are indicated with "DW" denoting dispersive waves. 

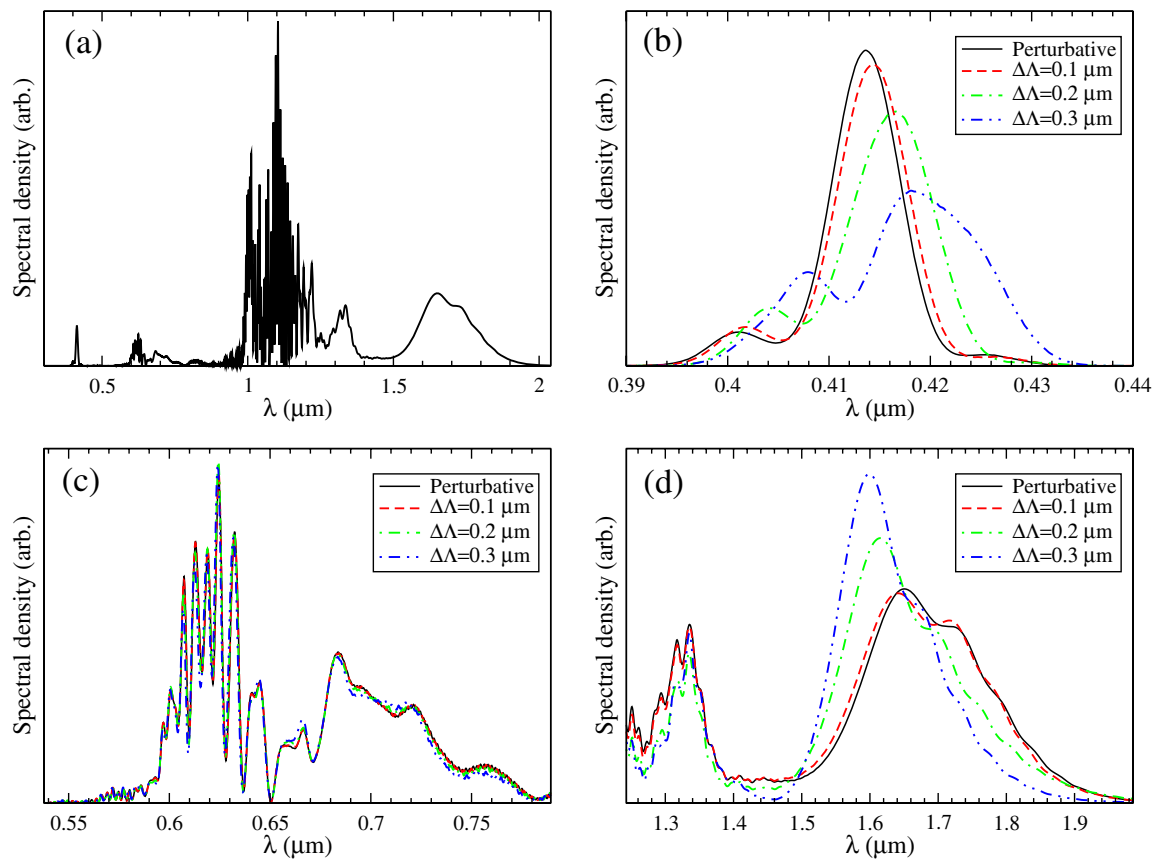

Fig. 6. (Color online) (a) Total spectrum of a $20 \mathrm{~kW} 50$ fs gaussian input pulse after a $5 \mathrm{~cm}$ taper from $\Lambda=4 \mu \mathrm{m}$ to $\Lambda=1 \mu \mathrm{m}$, with fiber parameters calculated along the taper by the perturbative approach. (b) Short-wavelength dispersive radiation, propagating behind main fundamental soliton, calculated either in the perturbative approach, or with linear interpolation between different $\Lambda$-values. (c) Intermediate-wavelength dispersive waves, propagating in front of soliton. (d) Spectrum of main soliton and long-wavelength dispersive waves.

and become well separated from it during propagation, as they propagate at a higher group velocity. However, a part of the dispersive waves are trapped behind the soliton, whose velocity is decreasing both from the Raman effect and the effect of the taper on dispersion, as discussed by $[8,26]$. As the dispersive wave "pushes" toward the trailing edge of the decelerating soliton, it is blue-shifted by cross-phase modulation. Toward the end of the taper, the soliton undergoes compression followed by spectral recoil and emission of longwavelength dispersive waves, as discussed above. The resulting spectrum is shown in Fig. 6(a). In Figs. 6(b) $-6(d)$, three portions of the spectrum, calculated with the different parameter interpolation methods, are compared. It is interesting to observe that the short- and long-wavelength parts of the spectrum are quite sensitive to a correct interpolation of the fiber parameters, whereas the dispersive-wave spectrum in the $600-800 \mathrm{~nm}$ range is more resilient. The long-wavelength spectrum is determined by the spectral recoil and dispersive-wave emission of the fundamental soliton, and is therefore highly sensitive to the exact placement of the longest zero-dispersion wavelength. The short-wavelength dispersive wave is shaped by cross-phase modulation with this soliton, and therefore highly sensitive to the group-velocity difference between the near-infrared and visible wavelengths. The intermediate-wavelength dispersive waves propagate in front of the main soliton, and have some interaction with solitonlike pulses around $1 \mu \mathrm{m}$, but these are weaker, and no "trapping" effect appears to be at work.

Further simulations, not shown here, indicated that it is mainly the final part of the taper, from $\Lambda=2 \mu \mathrm{m}$ to $\Lambda=1 \mu \mathrm{m}$, that needs to be accurately resolved for the resulting spectra to be accurate. While the requirements for accuracy clearly depend on the nature of the problem, a useful rule of thumb seems to be that $\Delta \Lambda / \Lambda$ should not exceed $10 \%$.

\section{CONCLUSION}

In conclusion, a full-vectorial nonlinear propagation formalism suitable for short broadband pulses in tapered optical fibers has been developed and tested. It has been demonstrated analytically and numerically that the formulation leads to photon-number conservation in a passive and lossless fiber model. A perturbative scheme for interpolating the dispersion properties of a tapered fiber along the taper was outlined, and compared to the simpler, and more general, scheme of linear interpolation between parameter sets calculated for fiber structures with different scales. For the latter case, it was shown numerically that good convergence requires the interpolation points to be separated by less than $100 \mathrm{~nm}$ in the overall length scale of the fiber.

\section{ACKNOWLEDGMENTS}

This work was financially supported by the Danish Council for Independent Research-Technology and Production Sciences (FTP).

\section{REFERENCES}

1. T. A. Birks, W. J. Wadsworth, and P. S. J. Russell, "Supercontinuum generation in tapered fibers," Opt. Lett. 25, 1415-1417 (2000).

2. L. Tong, R. R. Gattass, J. B. Ashcom, S. He, J. Lou, M. Shen, I. Maxwell, and E. Mazur, "Subwavelength-diameter silica wires for low-loss optical wave guiding," Nature 426, 816-819 (2003).

3. G. Brambilla, V. Finazzi, and D. Richardson, "Ultra-low-loss optical fiber nanotapers," Opt. Express 12, 2258-2263 (2004).

4. S. Leon-Saval, T. Birks, W. Wadsworth, P. S. J. Russell, and M. Mason, "Supercontinuum generation in submicron fibre waveguides," Opt. Express 12, 2864-2869 (2004).

5. M. A. Foster, J. M. Dudley, B. Kibler, Q. Cao, D. Lee, R. Trebino, and A. L. Gaeta, "Nonlinear pulse propagation and supercontinuum generation in photonic nanowires: experiment and simulation," Appl. Phys. B 81, 363-367 (2005). 
6. R. R. Gattass, G. T. Svacha, L. Tong, and E. Mazur, "Supercontinuum generation in submicrometer diameter silica fibers," Opt. Express 14, 9408-9414(2006).

7. A. Kudlinski, A. George, J. Knight, J. Travers, A. Rulkov, S. Popov, and J. Taylor, "Zero-dispersion wavelength decreasing photonic crystal fibers for ultraviolet-extended supercontinuum generation," Opt. Express 14, 5715-5722 (2006).

8. J. C. Travers and J. R. Taylor, "Soliton trapping of dispersive waves in tapered optical fibers," Opt. Lett. 34, 115-117 (2009).

9. A. Kudlinski, M. Lelek, B. Barviau, L. Audry, and A. Mussot, "Efficient blue conversion from a $1064 \mathrm{~nm}$ microchip laser in long photonic crystal fiber tapers for fluorescence microscopy," Opt. Express 18, 16640-16645 (2010).

10. S. T. Sørensen, A. Judge, C. L. Thomsen, and O. Bang, "Optimum fiber tapers for increasing the power in the blue edge of a supercontinuum-group-acceleration matching," Opt. Lett. 36, 816-818 (2011).

11. J. M. Dudley, G. Genty, and S. Coen, "Supercontinuum generation in photonic crystal fiber," Rev. Mod. Phys. 78, 1135-1184 (2006)

12. F. W. Wise, A. Chong, and W. H. Renninger, "High-energy femtosecond fiber lasers based on pulse propagation at normal dispersion," Laser Photon. Rev. 2, 58-73 (2008).

13. X. Liu, J. Lægsgaard, and D. Turchinovich, "Self-stabilization of a mode-locked femtosecond fiber laser using a photonic bandgap fiber," Opt. Lett. 35, 913-915 (2010).

14. X. Liu, J. Lægsgaard, and D. Turchinovich, "Highly-stable monolithic femtosecond yb-fiber laser system based on photonic crystal fibers," Opt. Express 18, 15475-15483 (2010).

15. X. Liu, J. Lægsgaard, U. Møller, H. Tu, S. A. Boppart, and D. Turchinovich, "All-fiber femtosecond Cherenkov radiation source," Opt. Lett. 37, 2769-2771 (2012).
16. O. Vanvincq, J. C. Travers, and A. Kudlinski, "Conservation of the photon number in the generalized nonlinear Schrödinger equation in axially varying optical fibers," Phys. Rev. A 84, 063820 (2011).

17. M. Kolesik, E. M. Wright, and J. V. Moloney, "Simulation of femtosecond pulse propagation in sub-micron diameter tapered fibers," Appl. Phys. B 79, 293-300 (2004).

18. S. A. Vahid and T. M. Monro, "A full vectorial model for pulse propagation in emerging waveguides with subwavelength structures part I: Kerr nonlinearity," Opt. Express 17, 2298-2318 (2009).

19. M. D. Turner, T. M. Monro, and S. A. Vahid, "A full vectorial model for pulse propagation in emerging waveguides with subwavelength structures part II: stimulated Raman scattering," Opt. Express 17, 11565-11581 (2009).

20. R. W. Hellwarth, "3rd-order optical susceptibilities of liquids and solids," Prog. Quantum Electron. 5, 1-68 (1977).

21. J. Lægsgaard, "Mode profile dispersion in the generalised nonlinear Schrödinger equation," Opt. Express 15, 16110-16123 (2007).

22. J. Lægsgaard and P. J. Roberts, "Dispersive pulse compression in hollow-core photonic bandgap fibers," Opt. Express 16, 9628-9644 (2008).

23. K. J. Blow and D. Wood, "Theoretical description of transient stimulated "Raman scattering in optical fibers," IEEE J. Quantum Electron. 25, 2665-2673 (1989).

24. G. P. Agrawal, Nonlinear Fiber Optics (Academic, 2007).

25. J. Lægsgaard, A. Bjarklev, and S. E. B. Libori, "Chromatic dispersion in photonic crystal fibers: fast and accurate scheme for calculation,” J. Opt. Soc. Am. B 20, 443-448 (2003).

26. A. V. Gorbach and D. V. Skryabin, "Theory of radiation trapping by the accelerating solitons in optical fibers," Phys. Rev. A 76, 053803 (2007). 